\title{
Prediction of dose-dependent in vivo acetylcholinesterase inhibition by profenofos in rats and humans using physiologically based kinetic (PBK) modeling-facilitated reverse dosimetry
}

\author{
Isaac Omwenga $^{1,2,4}$ D $\cdot$ Shensheng Zhao ${ }^{1} \cdot$ Laetitia Kanja $^{2} \cdot$ Hans Mol $^{3} \cdot$ Ivonne M. C. M. Rietjens $^{1} \cdot$ Jochem Louisse $^{3}$
}

Received: 7 September 2020 / Accepted: 16 February 2021 / Published online: 2 March 2021

(c) The Author(s) 2021

\begin{abstract}
Organophosphate pesticides (OPs) are known to inhibit acetylcholine esterase (AChE), a critical effect used to establish health-based guidance values. This study developed a combined in vitro-in silico approach to predict AChE inhibition by the OP profenofos in rats and humans. A physiologically based kinetic (PBK) model was developed for both species. Parameter values for profenofos conversion to 4-bromo-2-chlorophenol (BCP) were derived from in vitro incubations with liver microsomes, liver cytosol, and plasma from rats (catalytic efficiencies of 1.1, 2.8, and $0.19 \mathrm{ml} / \mathrm{min} / \mathrm{mg}$ protein, respectively) and humans (catalytic efficiencies of $0.17,0.79$, and $0.063 \mathrm{ml} / \mathrm{min} / \mathrm{mg}$ protein, respectively), whereas other chemical-related parameter values were derived using in silico calculations. The rat PBK model was evaluated against literature data on urinary excretion of conjugated BCP. Concentration-dependent inhibition of rat and human $\mathrm{AChE}$ was determined in vitro and these data were translated with the PBK models to predicted dose-dependent AChE inhibition in rats and humans in vivo. Comparing predicted dose-dependent $\mathrm{AChE}$ inhibition in rats to literature data on profenofos-induced AChE inhibition revealed an accurate prediction of in vivo effect levels. Comparison of rat predictions (BMDL10 of predicted dose-response data of $0.45 \mathrm{mg} / \mathrm{kg} \mathrm{bw}$ ) and human predictions (BMDL10 of predicted dose-response data of $0.01 \mathrm{mg} / \mathrm{kg}$ bw) suggests that humans are more sensitive than rats, being mainly due to differences in kinetics. Altogether, the results demonstrate that in vivo AChE inhibition upon acute exposure to profenofos was closely predicted in rats, indicating the potential of this novel approach method in chemical hazard assessment.
\end{abstract}

Keywords Organophosphate pesticides (OPs) - Physiologically based kinetic (PBK) modeling $\cdot$ Reverse dosimetry · Acetylcholinesterase (AChE) inhibition · Novel approach method (NAM)

Isaac Omwenga

isaac.omwenga@wur.nl

1 Division of Toxicology, Wageningen University and Research, Stippeneng 4, 6708 WE Wageningen, The Netherlands

2 Department of Public Health, Pharmacology and Toxicology, Faculty of Veterinary Sciences, University of Nairobi, P.O. Box 29053-00625, Nairobi, Kenya

3 Wageningen Food Safety Research, Wageningen University and Research, Akkermaalsbos 2, 6708 WB Wageningen, The Netherlands

4 Department of Animal Science, Meru University of Science and Technology, P.O. Box 972-60200, Meru, Kenya

\section{Introduction}

Organophosphorus or organophosphate pesticides (OPs) have been extensively used for the control of agricultural and household pests globally (Kumari and John 2019). Exposure to these pesticides has been reported for various segments of the population, including agriculture workers and their families, house hold members during home application of pesticides, people that live in proximity to farms, or the general public via residues on food (Bradman et al. 2003; Lu et al. 2000; Quandt et al. 2004). Consequently, OPs and their metabolites have been found in human blood, serum, urine, and breast milk (Liu et al. 2014; Hardt and Angerer 2000; Zhang et al. 2014; Naksen et al. 2016). Since various OPs have shown to cause adverse effects to the environment and to human health, many OPs are currently not registered 
for use as pesticides, and some OPs have even been banned (Hertz-Picciotto et al. 2018).

Epidemiological studies have linked chronic OP exposure to reproductive disorders, developmental toxicity, birth defects, cancer, Parkinson's disease, Alzheimer's disease, diabetes, chronic respiratory disease, cardiovascular diseases, chronic nephropathies and amyotrophic lateral sclerosis (ALS) (Mostafalou and Abdollahi 2013). The critical effects of OPs in animal studies are related to neurotoxicity, which has been reported to be related to, amongst others, inhibition of acetylcholinesterase (AChE), neuropathy target esterase (NTE) (Costa 2018), acylpeptide hydrolase (APH) (Richards et al. 2000), fatty acid amide hydrolase (FAAH) (Quistad et al. 2001; Buntyn et al. 2017), muscarinic M2 receptors (Costa 2006), and a variety of lipases (Quistad et al. 2006). Inhibition of AChE is one of the mechanisms by which OPs cause acute neurotoxicity (Jamal et al. 2002; Farahat et al. 2003), characterized by decreased hydrolysis of acetylcholine in both central and peripheral cholinergic synapses, resulting initially in overstimulation of nicotinic and muscarinic receptors, followed by receptor down-regulation on post-synaptic membranes (Costa 2006). Acute or repeated exposure to OPs can lead to organophosphate esterinduced delayed polyneuropathy (OPIDP), a neurodegenerative disorder caused by inhibition and aging of at least $70 \%$ of the activity of the neuropathy target esterase (NTE) present in nerve tissues as well as other tissues (e.g., lymphocytes, testis) (Johnson and Glynn 1995, 2001; Costa 2018). In the hazard and risk assessment of OPs, in vivo animal studies on OP-induced inhibition of AChE have been used to derive a point of departure (POD) to set safe exposure levels, such as an Acute Reference Dose (ARfD). ARfDs for chlorpyrifos, acephate, methamidofos, omethoate and profenofos as reported by organizations as the European Food Safety Authority (EFSA), United States Environmental Protection Agency (US EPA), and the Joint FAO/WHO Meeting on Pesticide Residues (JMPR) have been derived from data on OPinduced AChE inhibition from animal studies (JMPR 2007; EPA 2006, 2011, 2016; EFSA 2019). Although these in vivo studies do not measure complex neurotoxicity endpoints, the information on OP-induced inhibition of AChE in vivo is considered an important piece of information in the hazard characterization. In these in vivo studies, $\mathrm{AChE}$ activity is usually measured in blood and sometimes also brain tissue after OP exposure. In the present study, we aimed to assess whether in vivo dose-dependent OP-induced AChE inhibition can be predicted by an animal-free approach. To inhibit $\mathrm{AChE}$ in the in vivo situation, the OP needs to reach its target (AChE) at sufficiently high concentrations. The in vivo potency of an OP to inhibit AChE is, thus, dependent on its intrinsic ability (potency) to inhibit $\mathrm{AChE}$ and the amount of OP that reaches that target. The potency of an OP to inhibit AChE can be determined using in vitro approaches.
To estimate the amount of OP that reaches the target, socalled physiologically based kinetic (PBK) models can be used. A PBK model permits the simulation of the chemical's in vivo kinetics (ADME) and can relate external exposure to internal exposure at the target sites. When having PBK models for different exposure routes and/or different species, these models can be used for exposure route and/or species extrapolations. These models can also be used as a tool to estimate exposure applying reverse dosimetry of biomonitoring data (e.g., chemical levels in blood or urine), as has been shown, for example, for chlorpyrifos in rats and humans (Timchalk et al. 2002), and chlorpyrifos in children (Rigas et al. 2001). Furthermore, these models can be used for reverse dosimetry of in vitro toxicity data, thereby translating in vitro effect concentrations to in vivo doses, enabling prediction of in vivo dose-dependent toxicity, as has been shown for the OP chlorpyrifos (Zhao et al. 2019). Such PBK modeling-facilitated reverse dosimetry of in vitro toxicity data is considered crucial in the transition to nonanimal based novel approach methods (NAMs) for the safety assessment of chemicals (Louisse et al. 2017).

PBK models have been developed for various OPs including chlorpyrifos (Timchalk et al. 2002; Bouchard et al. 2005; Mosquin et al. 2009; Lu et al. 2010; Zhao et al. 2019), malathion (Bouchard et al. 2003, 2017; Bogen and Singhal 2017), parathion (Sultatos 1990) and diazinon (Poet et al. 2004). To date, no PBK model has been built for the OP profenofos, despite its widespread use in developing countries and reported cases of human accidental poisoning (Gotoh et al. 2001; Eddleston et al. 2009).

Profenofos [O-(4-bromo-2-chlorophenyl) $O$-ethyl $S$-propyl phosphorothioate] (Fig. 1) is a thiophosphate OP pesticide $(O=\mathrm{P}-\mathrm{S}-\mathrm{C})$ that was developed for pest strains resistant to chlorpyrifos and other OPs (Gotoh et al. 2001). Profenofos has been classified as a moderately hazardous (Toxicity Class II) pesticide by the World Health Organization (WHO) with moderate level of acute toxicity (LD50 of $358-1178 \mathrm{mg} / \mathrm{kg}$ in rat) following oral administration (Reported in JMPR (2007)). Dietary intake of profenofos is the primary exposure route for humans (Greish et al. 2011) and residue levels exceeding EU MRLs have been found in various vegetables in Kenya (Karanja et al. 2012). Our recent study reported profenofos as one of the most frequently encountered pesticide residues in vegetables sampled in peri-urban Nairobi, Kenya (Omwenga et al. 2021).

The present study aims to develop a PBK model for profenofos in rats and humans and apply the models to predict dose-dependent in vivo AChE inhibition using PBK modeling-facilitated reverse dosimetry of in vitro data on profenofos-induced AChE inhibition, allowing interspecies comparisons and providing a proof-of-principle that OPinduced AChE inhibition can be predicted for both rats and humans without the need for in vivo studies. To that end, 


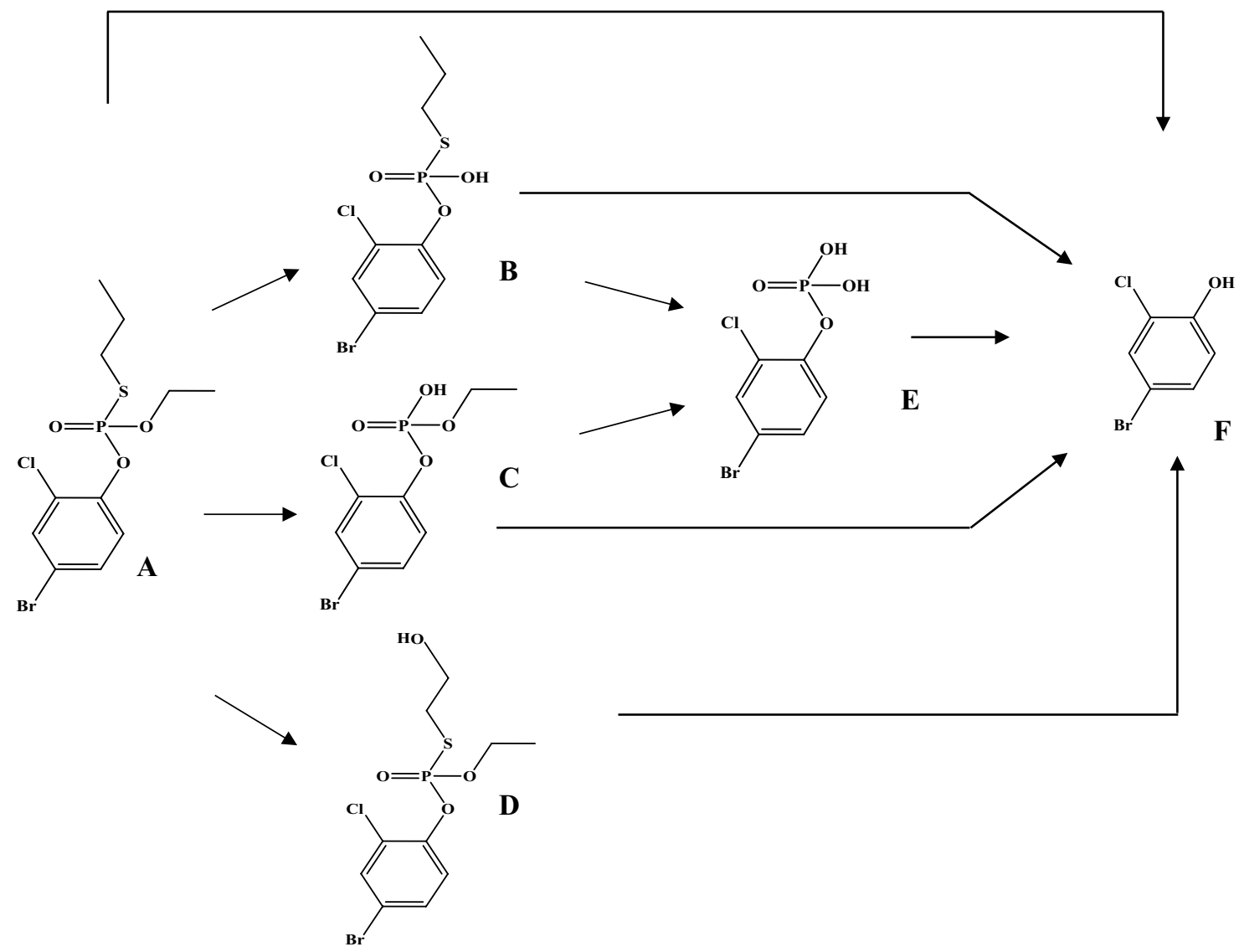

Fig. 1 Metabolic pathways for metabolism of profenofos (a) based on data reported in previous studies (Gotoh et al. 2001; Dadson et al. 2013). Metabolites include desethylated profenofos (b), desthiopro-

profenofos PBK models were developed based on in silicoand in vitro-derived input parameter values. The rat PBK model was evaluated by comparing PBK model predictions with available in vivo kinetic data in rats. Subsequently, in vitro data on profenofos-induced $\mathrm{AChE}$ inhibition were translated to predicted in vivo dose-dependent AChE inhibition in rats and humans, and predictions on dose-dependent $\mathrm{AChE}$ inhibition in rats were evaluated by comparison with available in vivo data.

\section{Materials and methods}

\section{Materials}

Profenofos, 4-bromo-2-chlorophenol (BCP), bovine serum albumin (BSA), reduced nicotinamide adenine dinucleotide phosphate (NADPH), 5,5-dithiobis (2-nitrobenzoic acid) (DTNB) and acetylthiocholine iodide (ATC) were purchased from Sigma-Aldrich (Zwijndrecht, The Netherlands). Magnesium chloride hexahydrate $\left(\mathrm{MgCl}_{2} \cdot 6 \mathrm{H}_{2} \mathrm{O}\right)$, trifluoroacetic acid (TFA), dimethylsulfoxide (DMSO), and
pylprofenofos/des-S-propylated profenofos (c), hydroxypropyl profenofos (d), 4-bromo-2-chlorophenyl dihydrogen phosphate (e), and 4-bromo-2-chlorophenol (BCP) (f)

calcium chloride dihydrate $\left(\mathrm{CaCl}_{2} \cdot 2 \mathrm{H}_{2} \mathrm{O}\right)$ were purchased from VWR International (Amsterdam, The Netherlands). Acetonitrile (ACN, UPLC/MS grade) was purchased from Biosolve (Valkenswaard, The Netherlands). Human liver microsomes and cytosol (pooled from 150 human donors, mixed gender), rat liver microsomes and rat liver cytosol (pooled from 20 male Sprague-Dawley rats) were purchased from Corning (Amsterdam, The Netherlands). Recombinant human acetylcholinesterase was purchased from SigmaAldrich (Zwijndrecht, The Netherlands). Rat blood was purchased from BioIVT (Westbury, USA), human and rat plasma were purchased from Innovate (New York, USA).

\section{PBK model development}

\section{Model structure and software}

The PBK model structure applied (Fig. 2) is based on a generic PBK model that has been developed for humans (Jones and Rowland-Yeo 2013). Physiological parameter values (tissue volumes and blood flows) for rats and humans were taken from Jones and Rowland-Yeo (2013) 


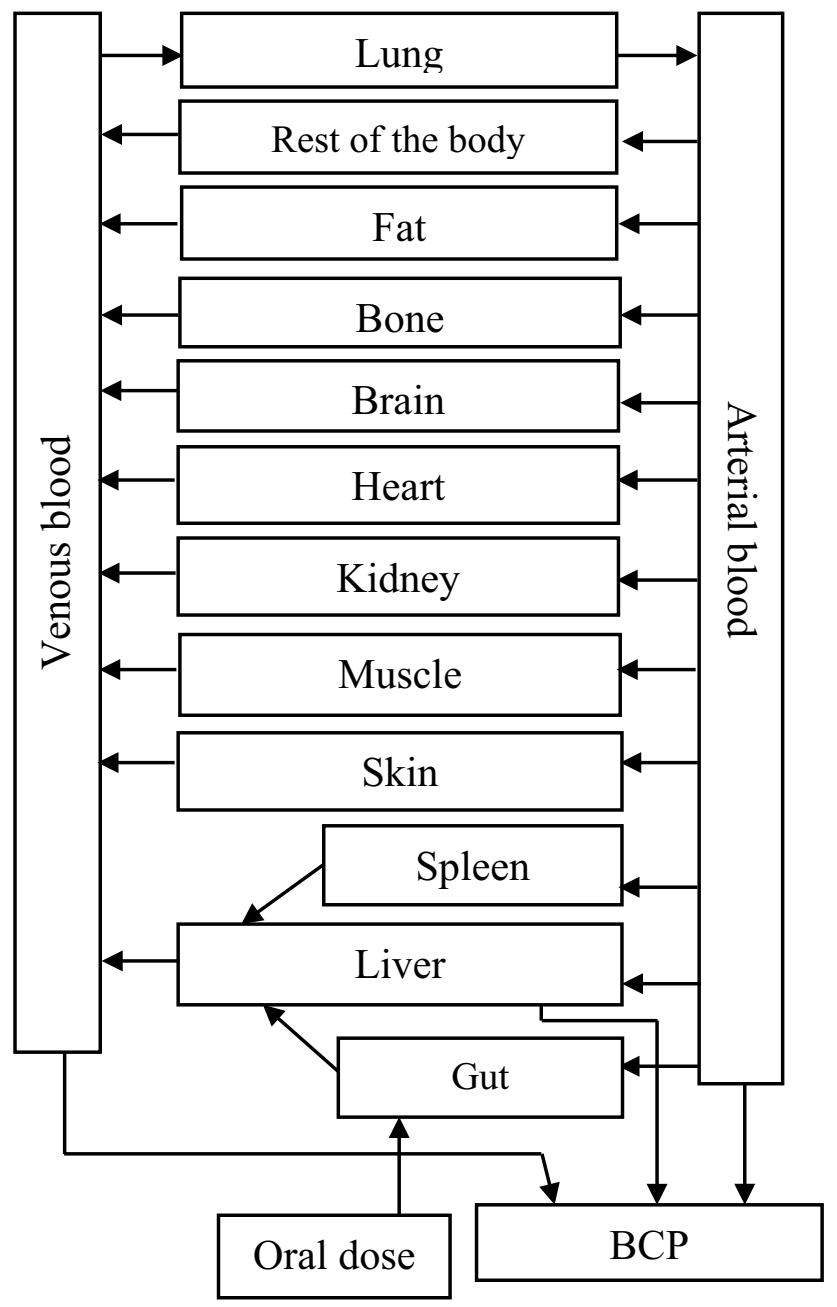

Fig. 2 Schematic diagram of the PBK model for profenofos for rats and humans

(Supplementary Table 1) as collected previously by Punt et al. (2020). The generic model contains separate compartments for liver, the gastrointestinal tract (GI-tract), fat, muscle, skin, bone, brain, heart, kidney, lung, spleen, venous blood, arterial blood, and a rest-of-body compartment. The uptake of profenofos from the GI-tract was described as a first-order process with an absorption rate constant of $1 \mathrm{~h}^{-1}$. All absorbed profenofos was assumed to be transferred directly to the liver compartment via the portal vein. Tissue:plasma partition coefficients were estimated based on the method of Berezhkovskiy (2004) using $\log K_{o w}$, pKa and fraction unbound plasma (fup) as input parameters. $\log K_{\text {ow }}$ and $\mathrm{pKa}$ values were estimated using chemicalize ( $\mathrm{www}$. chemicalize.com) and fup was estimated (based on $\log \mathrm{P}$ and pKa) using the online simcyp tool (https://www.certara.com/ software/pbpk-modeling-and-simulation/).

The main metabolite of profenofos reported in vivo is 4-bromo-2-chlorophenol (BCP), which has been reported to be formed upon paraoxonase 1 (PON1)-mediated hydrolysis of profenofos (JMPR 2007; EPA 2016; Dadson et al. 2013) (Fig. 1). Profenofos hydrolysis (BCP formation) was quantified in the present study using rat and human liver microsomes, liver cytosol and plasma (In vitro incubations to derive kinetic parameter values for description of metabolic clearance in the PBK model). Profenofos clearance was included in the blood and liver compartments of the PBK model. In in vitro studies, also CYP-mediated formation of other metabolites has been reported (in the presence of inhibitors of hydrolysis enzymes) (Dadson et al. 2013), but these are considered less relevant, since the hydrolysis reaction is more efficient than the CYP-mediated oxidations, as indicated in our in vitro metabolism studies with liver microsomes or cytosol in which practically all profenofos was converted into BCP. Since BCP was shown to not inhibit AChE (see "Results"), only profenofos kinetics were considered relevant for the reverse dosimetry and included in the PBK model (Fig. 2). The only in vivo data publicly available on profenofos kinetics in rats or humans, required for model evaluation, relate to the urinary excretion of conjugated BCP in rats. Therefore, to allow model evaluation, the rat PBK model was extended to include a sub-model on BCP and BCP-glucuronide formation (Supplementary Fig. 1). Estimated tissue:plasma partition coefficients of BCP and BCP-glucuronide and fraction unbound plasma (fup) values used for these sub-models are presented in Supplementary Table 1.

Renal excretion is also included in the model which is described by a passive renal clearance (via glomerular filtration) of the unbound fraction in blood at the rate of $6.7 \mathrm{~L} \mathrm{~h}^{-1}$ in humans and $0.078 \mathrm{~L} \mathrm{~h}^{-1}$ in rats. The model equations were coded and numerically integrated in Berkeley Madonna 9.1.18 (UC Berkeley, CA, USA), using the Rosenbrock's algorithm for stiff systems. The PBK models' differential equations are provided in the Supplementary Materials.

\section{In vitro incubations to derive kinetic parameter values for description of metabolic clearance in the PBK model}

Incubations of profenofos with rat and human liver microsomes, liver cytosol and plasma were performed to quantify in vitro rates of $\mathrm{BCP}$ formation. Conditions were optimized to be linear for metabolite formation with regard to incubation time and microsomal, cytosolic and plasma protein concentration (data not shown). The final incubations were

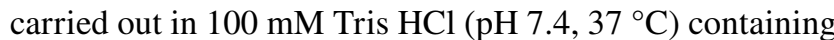
(final concentrations) $5 \mathrm{mM} \mathrm{MgCl}_{2}, 2 \mathrm{mM} \mathrm{CaCl}_{2}$ [to stimulate PON1 activity (Carr et al. 2015)], 2 mM NADPH (cofactor to also include CYP-mediated metabolism), enzyme preparation (final concentration $2 \mathrm{mg} / \mathrm{ml}$ for human liver microsomes and cytosol and $4.4 \mathrm{mg} / \mathrm{ml}$ for human plasma; $0.5 \mathrm{mg} / \mathrm{ml}$ for rat microsomes and cytosol and $1.65 \mathrm{mg} /$ $\mathrm{ml}$ for rat plasma) and profenofos (at final concentrations 
ranging from 1 to $100 \mu \mathrm{M}$, added from 100 times concentrated stock solutions in DMSO). Control incubations were performed in the absence of microsomes, cytosol or plasma. After 2 min pre-incubation, the reaction was initiated by adding the substrate and mixtures were incubated for $2 \mathrm{~min}$ in a $37{ }^{\circ} \mathrm{C}$ water bath. The total volume of the incubation mixtures was $200 \mu \mathrm{l}$. The reaction was terminated by the addition of $50 \mu \mathrm{l}$ ice cold ACN and samples were kept on ice. The mixture was centrifuged at $14,000 \mathrm{~g}$ for $20 \mathrm{~min}$ at $4{ }^{\circ} \mathrm{C}$ and the supernatant was analyzed using UPLC-UV.

\section{UPLC-UV analysis}

All samples from incubations were analyzed using a Waters Acquity UPLC $\mathrm{H}$ class system that consisted of a quaternary solvent manager, a sample manager, and a photodiode array (PDA) detector, equipped with a Water Acquity UPLC $^{\circledR}$ BEH C18 column $(1.7 \mu \mathrm{m}, 2.1 \times 50 \mu \mathrm{mm})$ and Waters Xbridge UPLC ${ }^{\circledR}$ BEH C18 pre-column $(2.5 \mu \mathrm{M}$, $2.1 \times 5 \mathrm{~mm}$ ). The temperature of the column was kept at $40{ }^{\circ} \mathrm{C}$ and the auto sampler at $10{ }^{\circ} \mathrm{C}$ during the UPLC analysis. The mobile phases used for the analysis consisted of (A) $0.1 \%$ TFA in nanopure water and (B) $100 \%$ ACN. A gradient elution at a flow rate of $0.6 \mathrm{ml} / \mathrm{min}$ was applied for the analysis with the initial condition of 100\% A:0\% B (v/v), changing in a linear way to $0 \% \mathrm{~A}: 100 \% \mathrm{~B}$ from 0 to $6 \mathrm{~min}$, which was maintained for $30 \mathrm{~s}$, and then changed back to the initial conditions in $30 \mathrm{~s}$, which were maintained for $1 \mathrm{~min}$. The injection volume for each sample was $3.5 \mu 1$.

Under these conditions, the retention times of profenofos and BCP were 4.76 and $3.37 \mathrm{~min}$, respectively. The amounts of profenofos and $\mathrm{BCP}$ were quantified by integrating the peak areas at $237 \mathrm{~nm}$ using calibration curves that were prepared using commercially available standards.

\section{In vitro metabolism data analysis and scaling in the PBK model}

Kinetic parameters including the apparent maximum velocity (Vmax) and the apparent Michaelis-Menten constant $(\mathrm{Km})$ for BCP formation were obtained by fitting the data for the substrate concentration-dependent rate of conversion (expressed in $\mathrm{nmol} / \mathrm{min} / \mathrm{mg}$ protein) using GraphPad Prism 5, version 5.04 (San Diego, California, USA) to the standard Michaelis-Menten equation:

$V=V_{\max } *[S] /(\mathrm{Km}+[S])$

in which the $S$ represents the concentration of substrate, expressed in $\mu \mathrm{M}, V$ and $V_{\max }$ the velocity and the maximum velocity of the reaction, respectively, expressed in $\mathrm{nmol} /$ $\mathrm{min} / \mathrm{mg}$ protein, and $\mathrm{Km}$ the apparent Michaelis-Menten constant, expressed in $\mu \mathrm{M}$. The kinetic parameter values for conversion of profenofos to $\mathrm{BCP}$ in the liver microsomes, liver cytosol and plasma were determined. To determine the catalytic efficiency, $V_{\max }$ was divided by the $\mathrm{Km}$.

The in vitro $V_{\max }$ values were scaled in the PBK model code (Supplementary Materials) using the following scaling factors for rats and humans: $35 \mathrm{mg}$ microsomal protein $/ \mathrm{g}$ liver, $80.7 \mathrm{mg}$ cytosolic protein/g liver and $550 \mathrm{mg}$ plasma/g blood (Medinsky et al. 1994; Cubitt et al. 2011). The apparent Vmax values obtained from enzymatic incubations expressed in $\mathrm{nmol} / \mathrm{min} / \mathrm{mg}$ protein were converted into $\mu \mathrm{mol} / \mathrm{h} / \mathrm{kg}$ liver and plasma in the PBK model code. The in vivo $\mathrm{Km}$ values were assumed to be equal to those obtained in vitro (taking into account the differences in free fraction in vitro vs in vivo).

\section{Model evaluation}

Since the only in vivo data available on profenofos kinetics for model evaluation were on the urinary excretion of conjugated $\mathrm{BCP}$ in rats, the rat PBK model was extended to include a sub-model on BCP and BCP-glucuronide (Supplementary Fig. 1). Estimated partition coefficients and fup values of $\mathrm{BCP}$ and $\mathrm{BCP}$-glucuronide used for these submodels are presented in Supplementary Table 1 and conversion of $\mathrm{BCP}$ to $\mathrm{BCP}$-glucuronide was assumed to take place only in the liver having the same apparent $\mathrm{Km}$ and $V_{\max }$ values as reported before by Strikwold et al. (2013) for phenol glucuronidation. The PBK model-predicted cumulative urinary excretion of BCP-glucuronide were compared with reported in vivo data on conjugated $\mathrm{BCP}$ excretion in profenofos-exposed rats (Cho et al. 2002).

Furthermore, a sensitivity analysis was performed to estimate the impact of the chemical-specific parameters on the model output [maximum free (unbound) blood concentrations of profenofos in this study]. Normalized sensitivity coefficients (SCs) were calculated using the following equation:

$\mathrm{SC}=\left(C^{\prime}-C\right) /\left(P^{\prime}-P\right) \times(P / C)$.

in which $P$ represents the parameter value in the PBK model and $P^{\prime}$ the parameter value with a 5\% increase (Evans and Andersen 2000). Likewise, $C^{\prime}$ represents the model output obtained with the 5\% increase in $P$, while $C$ is the model output using the initial model parameter value. The sensitivity analysis was conducted for oral exposure to predicted effect doses obtained with the reverse dosimetry analysis (BMDL 10 values). $\mathrm{BMDL}_{10}$ values were selected since a $\mathrm{BMDL}_{10}$ value from a rat study on profenofos-induced $\mathrm{AChE}$ inhibition was recently used to derive the POD to obtain an acute reference dose (ARfD) for profenofos by the US EPA (EPA 2016). 


\section{Determination of in vitro AChE inhibition by profenofos and quantitative in vitro to in vivo extrapolation (QIVIVE) using reverse dosimetry}

To perform reverse dosimetry of in vitro $\mathrm{AChE}$ inhibition by profenofos, first the in vitro AChE activity in the absence or presence of increasing concentrations of profenofos was determined. For humans, commercially available recombinant AChE was used. For rats, no commercially available recombinant $\mathrm{AChE}$ was available, so rat $\mathrm{AChE}$ was obtained from rat blood as described below.

\section{Derivation of a concentration-response curve for human recombinant $\mathrm{AChE}$ inhibition}

AChE inhibition was determined following the protocol of Ellman et al. (1961) as modified by Chambers and Chambers (1989). The concentration of acetylthiocholine and recombinant AChE were optimized to be linear for metabolite formation (data not shown). Human recombinant AChE was dissolved in $0.1 \mathrm{M}$ sodium phosphate ( $\mathrm{pH}$ 7.4) containing $1 \mathrm{mg} / \mathrm{ml} \mathrm{BSA} \mathrm{(which} \mathrm{was} \mathrm{present} \mathrm{to} \mathrm{stabilize} \mathrm{human} \mathrm{recom-}$ binant AChE (Rosenfeld and Sultatos 2006)) to reach an enzyme concentration of $200 \mathrm{U} / \mathrm{ml}$. Subsequently, the AChE enzyme solution was further diluted with $0.1 \mathrm{M}$ sodium phosphate ( $\mathrm{pH}$ 7.4) containing $1 \mathrm{mg} / \mathrm{ml}$ BSA to a working concentration of $1 \mathrm{U} / \mathrm{ml}$. A range of concentrated stock solutions of profenofos were prepared in ethanol and 50 times diluted by adding $5 \mu \mathrm{l}$ of stock solution to $245 \mu \mathrm{l}$ of $0.1 \mathrm{M}$ sodium phosphate ( $\mathrm{pH} 7.4$ ) (containing $0.1 \mathrm{mg} / \mathrm{ml} \mathrm{BSA}$ ). For the negative control, $5 \mu \mathrm{l}$ of ethanol without profenofos was added to $245 \mu \mathrm{l}$ of $0.1 \mathrm{M}$ sodium phosphate ( $\mathrm{pH} 7.4$ ) (containing $0.1 \mathrm{mg} / \mathrm{ml} \mathrm{BSA}$ ). Subsequently, $5 \mu \mathrm{l}$ of these working solutions was added into wells of a 96-well plate already containing 44- $\mu$ l sodium phosphate $(\mathrm{pH} 7.4)$ (containing $0.1 \mathrm{mg} / \mathrm{ml} \mathrm{BSA}$ ). To start the reaction (in absence or presence of a range of profenofos concentrations), 1- $\mu$ l enzyme solution was added, giving a total volume of $50 \mu \mathrm{l}$ per reaction with an AChE concentration of $0.02 \mathrm{U} / \mathrm{ml}$ and an ethanol concentration of $0.2 \%$, a level that had no effect on the activity of the enzyme. After 15-min incubation at $37{ }^{\circ} \mathrm{C}$, the reactions were stopped by adding $150 \mu \mathrm{l}$ of a mixture of 5,5-dithiobis (2-nitrobenzoic acid) (DTNB) and acetylthiocholine (ATC) (final DTNB and ATC concentrations were 0.075 and $0.15 \mathrm{mM}$, respectively). Subsequently, the time-dependent increase in absorbance due to formation of chromophore (DTNB + thiocholine) was measured at $37{ }^{\circ} \mathrm{C}$ using a wavelength of $412 \mathrm{~nm}$ during a period of $10 \mathrm{~min}$, using a spectrophotometer (SpectraMax, Molecular Devices, UK).

AChE activity was expressed as enzyme activity (percent of control). The concentration of profenofos that produced a $50 \%$ decrease in AChE activity (IC50) was determined from best-fit plots of the mean $( \pm S D)$ percentages of inhibition vs. the $10 \log \log$ arithm of profenofos concentrations using GraphPad Prism 5, version 5.04 (San Diego, California, USA) equation:

$Y=100 /\left(1+10^{(X-\log I C 50)}\right)$.

\section{Derivation of a concentration-response curve for rat erythrocyte AChE inhibition}

Blood sample processing and enzyme extraction Rat blood samples were processed according to a method reported by Larsen et al. (2019) with a few modifications to isolate the extrinsic membrane bound AChE. Briefly, the blood sample $(2 \mathrm{ml})$ was centrifuged at $3000 \mathrm{~g}$ for $15 \mathrm{~min}$ to separate plasma and cells. Aliquots of $500 \mu \mathrm{l}$ of the cells were resuspended in $4.5 \mathrm{ml}$ lysis buffer $(20 \mathrm{mM}$ sodium phosphate; $\mathrm{pH} 7.4$ ) and frozen at $-80{ }^{\circ} \mathrm{C}$ for $24 \mathrm{~h}$. After that, the cell samples were thawed and centrifuged (4000g) for $15 \mathrm{~min}$ at $4{ }^{\circ} \mathrm{C}$. The supernatant was poured off and the precipitate was suspended in 4.5-ml lysis buffer. This treatment was repeated another two times. The residue containing what is called erythrocyte 'ghost' membranes, was resuspended in $500-\mu \mathrm{l}$ analysis buffer (100 mM sodium phosphate; $\mathrm{pH} 7.4$ ), and stored at $-80{ }^{\circ} \mathrm{C}$ until analysis. Aliquots of erythrocyte 'ghost' membrane preparations were used for protein measurement using the Pierce ${ }^{\mathrm{TM}}$ BCA Protein Assay Kit (Thermofisher) with BSA as standard for quantification (Lowry et al. 1951), and for AChE activity assessment.

Rat red blood cell (RBC) AChE activity The effect of profenofos on rat AChE activity was assessed as described above for human recombinant $\mathrm{AChE}$, with a few modifications. Briefly, a typical reaction mixture $(200 \mu \mathrm{l})$ for AChE activity contained $0.005 \mathrm{mg} / \mathrm{ml}$ erythrocyte 'ghost' membrane protein. The rest of the steps were as described above for measurement of human recombinant AChE activity.

\section{QIVIVE of AChE inhibition data with PBK modeling-facilitated reverse dosimetry}

In the present study, it was assumed that in vivo dose-dependent AChE inhibition (in blood) depends on the maximum concentration $\left(C_{\max }\right)$ of profenofos reached in the blood. For adequate PBK modeling-facilitated reverse dosimetry, the active (unbound) concentration of a test chemical in the in vitro test system should be linked to the in vivo freely available chemical at the target site. This is important since it is assumed that it is the fraction unbound that causes the effect (AChE inhibition). In the in vitro incubations, a very low concentration of BSA $(0.1 \mathrm{mg} / \mathrm{mL})$ is present. Heilmair et al. (2008) reported that with such low BSA concentrations, the free concentration 
of chlorpyrifos oxon is not affected. Furthermore, Rosenfeld and Sultatos (2006) found no evidence of binding of paraoxon by BSA even at a higher concentration $(1 \mathrm{mg} / \mathrm{ml})$ during incubations. Based on these observations, no significant effect on the free fraction of profenofos in the in vitro $\mathrm{AChE}$ inhibition studies is expected, which corroborated with estimations on profenofos binding in the in vitro incubations with help of the online simcyp tool (https://www.certara.com/software/ pbpk-modeling-and-simulation/). A description of the fraction unbound in blood is incorporated in the PBK model, hence, the predicted in vivo unbound $C_{\max }$ values in blood were related to the profenofos concentrations we applied in vitro.

By calculating with the PBK model, the external dose required to reach (as unbound $C_{\max }$ ) the concentrations applied in the in vitro test, each in vitro concentration was translated into an in vivo dose. In this way, the concentration-response curves for rat and human AChE inhibition were converted into in vivo dose-response curves for profenofos-induced AChE inhibition in rats and humans, respectively.

\section{Evaluation of predicted dose-response curves for rat and human AChE inhibition}

To evaluate the performance of the PBK modeling-facilitated reverse dosimetry approach to predict in vivo $\mathrm{AChE}$ inhibition, the predicted dose-response curve for rat $\mathrm{AChE}$ inhibition upon exposure to profenofos was compared with available in vivo data on AChE inhibition in rats (JMPR 2007). Furthermore, the predicted dose-response data were used for BMD modeling, using EFSA PROAST version 69.0 (https://shiny-efsa.openanalytics.eu/app/bmd) using the model averaging approach to allow evaluation of the prediction by comparison of $\mathrm{BMDL}_{10}$ values obtained from the predicted dose-response data to points of departure derived by regulatory bodies (EPA 2016; JMPR 2007, EFSA 2019). To that end, the obtained $\mathrm{BMDL}_{10}$ values were compared with a reported $\mathrm{BMDL}_{10}$ value from a rat study on profenofos-induced RBC AChE inhibition, which was recently used to obtain an ARfD by the US EPA (EPA 2016), and a reported NOAEL value on profenofos-induced rat brain acetylcholinesterase inhibition, which has been used to obtain an ARfD by JMPR (2007).

\section{Results}

\section{In vitro conversion of profenofos to BCP}

The conversion of profenofos to BCP was measured in incubations with both rat and human liver microsomes, cytosol and plasma. UPLC analysis revealed that only one peak (BCP) appeared when analyzing the samples obtained from these incubations. In control incubations, small amounts (max $0.04 \%$ of the amount of profenofos added) of BCP were detected, indicating some spontaneous hydrolysis of profenofos in the aqueous environment, which has also been observed in previous studies (Aly and Badawy 1982). The $\mathrm{BCP}$ formation data for microsomes, cytosol and plasma were, therefore, corrected for BCP levels detected in control incubations.

The concentration-dependent velocity of $\mathrm{BCP}$ formation following incubation of profenofos with both human and rat liver microsomes, liver cytosol and plasma is depicted in Fig. 3a-c. The kinetic parameters derived from these data $\left(\mathrm{Km}\right.$ and $\left.V_{\max }\right)$ as well as the catalytic efficiencies, calculated as $V_{\max } / \mathrm{Km}$, are presented in Table 1 .

The results indicate differences in the catalytic efficiencies for conversion of profenofos to BCP by rats and humans (Table 1). The catalytic efficiency for microsomal biotransformation of profenofos to BCP was 6.5-fold lower in incubations with human liver microsomes than with rat liver microsomes, due to a 7.7-fold lower Vmax and a 1.2-fold lower $\mathrm{Km}$ (Table 1). Likewise, the catalytic efficiency for cytosolic biotransformation of profenofos to BCP was 3.8fold lower for human liver cytosol, with a 1.1-fold higher $V_{\text {max }}$, and a 4.1-fold higher $\mathrm{Km}$ for human liver cytosol as compared to rat liver cytosol. Largest differences were observed for plasma, indicated by the 32-fold lower catalytic efficiency of biotransformation of profenofos to BCP for human plasma than for rat plasma, due to a 1.5-fold higher $V_{\max }$ and 44-fold higher $\mathrm{Km}$ for human plasma as compared to rat plasma.

\section{PBK model development and evaluation}

Using the kinetic parameters defined in vitro and the input parameters obtained with in silico methods summarized in Supplementary Table 1, PBK models for profenofos in rat and human were made. Figure 4 presents the rat PBK modelbased prediction of the urinary BCP excretion. In a subsequent step, first, the developed rat profenofos PBK model was evaluated against in vivo data on urinary excretion of conjugated BCP in rats from a study by Cho et al. (2002). These results indicate that the predictions made by the newly developed PBK model for rat match the reported in vivo data well. Due to a lack of in vivo kinetic data for humans, evaluation of the performance of the PBK model for profenofos in humans could not be performed. However, the human model was considered to be adequate, since it is based on the same conceptual model and in vitro- and in silico-derived input parameters were defined in the same way as those for the rat model.

Further evaluation of the PBK models included a local sensitivity analysis in which the impact of each chemicalspecific parameter on the predicted free (unbound) $C_{\max }$ 

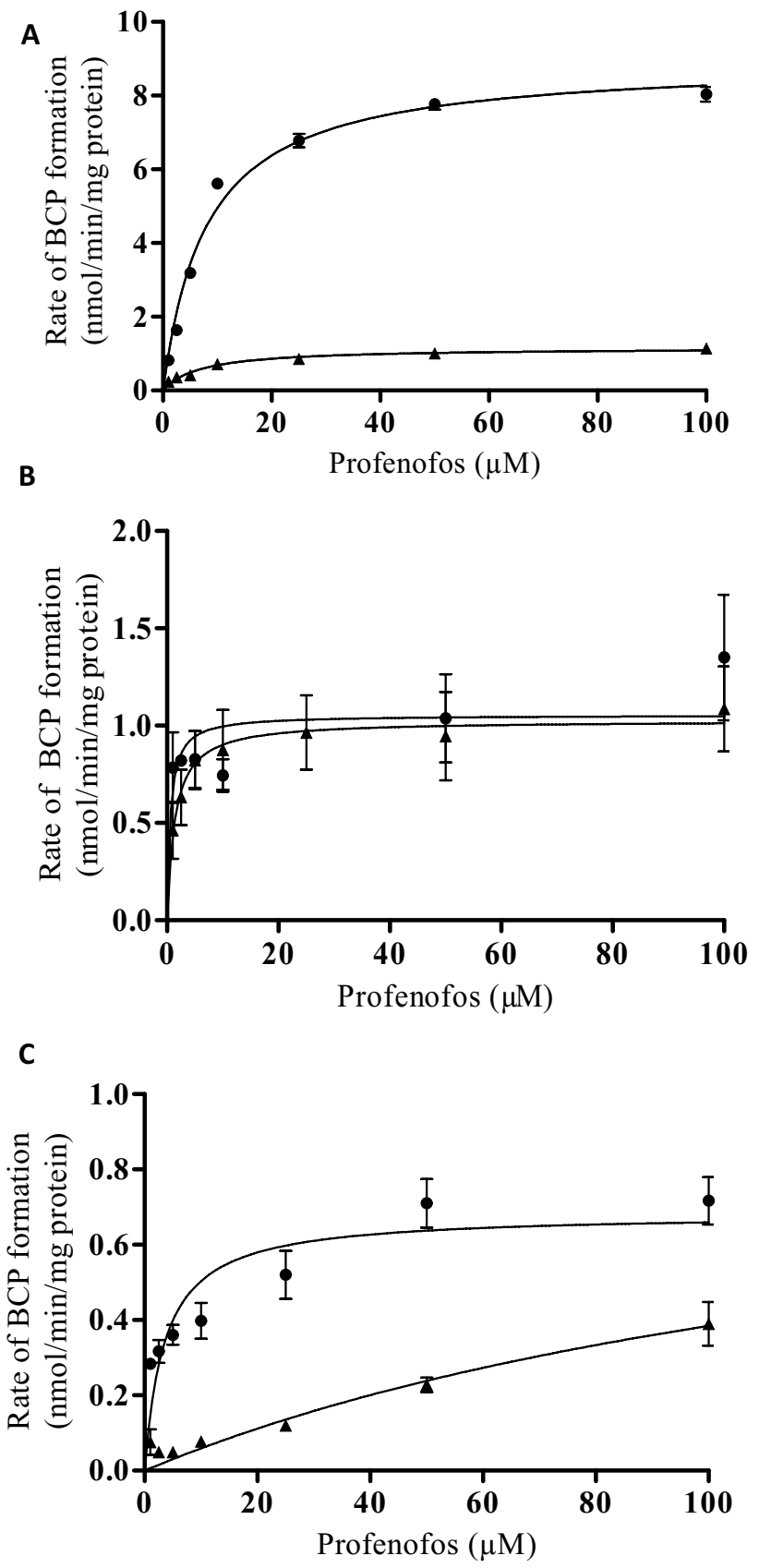

Fig. 3 Concentration-dependent rate of profenofos conversion to $\mathrm{BCP}$ in incubations with human (triangles) and rat (dots) a liver microsomal proteins, b liver cytosolic proteins, and $\mathbf{c}$ plasma proteins. Results represent data from 3 independent experiments and are presented as mean \pm SEM

of profenofos in the rat and human PBK model was determined upon exposure to predicted effect doses $\left(\mathrm{BMDL}_{10}\right.$ values derived from predicted dose-response data). Figure 5 presents the parameters for which the SCs are higher than 0.1 (absolute value). For both rat and human models, the prediction of the $C_{\max }$ of profenofos appeared to be
Table 1 Kinetic parameter values $\left(\mathrm{Km}, V_{\max }\right)$ and catalytic efficiencies $\left(V_{\max } / \mathrm{Km}\right)$ for in vitro conversion of profenofos to BCP

\begin{tabular}{lll}
\hline & Human & Rat \\
\hline Liver microsomes & & \\
Km $(\mu \mathrm{M})$ & 6.9 & 8.1 \\
$V_{\text {max }}(\mathrm{nmol} / \mathrm{min} / \mathrm{mg}$ microsomal protein) & 1.2 & 8.9 \\
Catalytic efficiency (ml/min/mg protein) & 0.17 & 1.1 \\
Liver cytosol & & \\
Km $(\mu \mathrm{M})$ & 1.4 & 0.34 \\
$V_{\max }(\mathrm{nmol} / \mathrm{min} / \mathrm{mg}$ cytosolic protein) & 1.1 & 0.95 \\
Catalytic efficiency (ml/min/mg protein) & 0.79 & 2.8 \\
Plasma & & \\
Km ( $\mu \mathrm{M})$ & 158 & 3.6 \\
$V_{\text {max }}(\mathrm{nmol} / \mathrm{min} / \mathrm{mg}$ plasma protein) & 0.99 & 0.68 \\
Catalytic efficiency (ml/min/mg protein) & 0.0063 & 0.19 \\
\hline
\end{tabular}

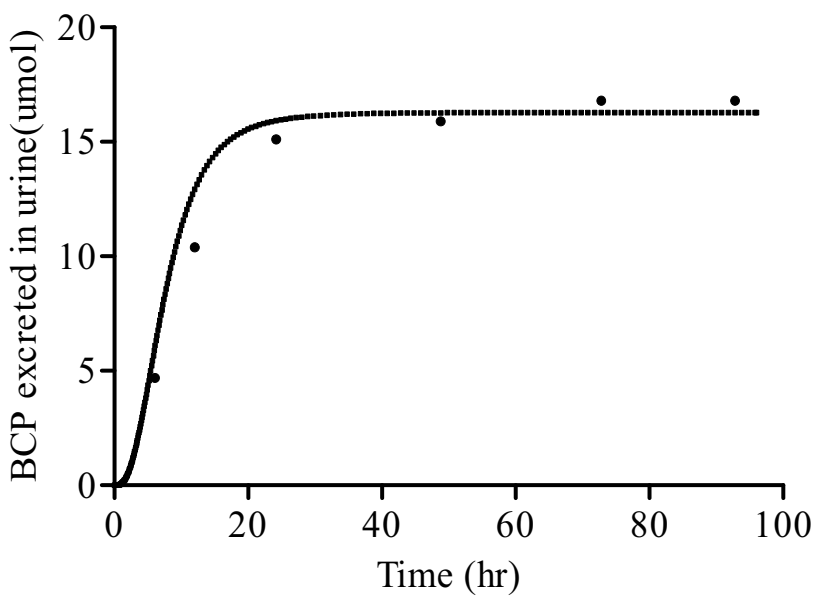

Fig. 4 Comparison of PBK model-predicted (continuous line) and experimentally determined (symbols, Cho et al. 2002) time-dependent cumulative urinary excretion of BCP-glucuronide in rats upon oral administration of $35.8 \mathrm{mg}$ profenofos $/ \mathrm{kg}$ bw

mainly affected by the kinetic parameters for conversion of profenofos to BCP by enzymes from especially plasma and liver cytosol, in addition to the absorption rate constant (ka) and the gut to plasma partition coefficient.

The obtained PBK models reveal differences in kinetics in rats and humans (Fig. 6). Figure 6 shows the PBK model-predicted dose-dependent $C_{\max }$ values (free concentration) in rats and humans, indicating that humans are expected to reach around 20-fold higher blood concentrations than rats at equal oral exposure levels, mainly due to the lower catalytic efficiency for metabolic clearance of profenofos in humans. 


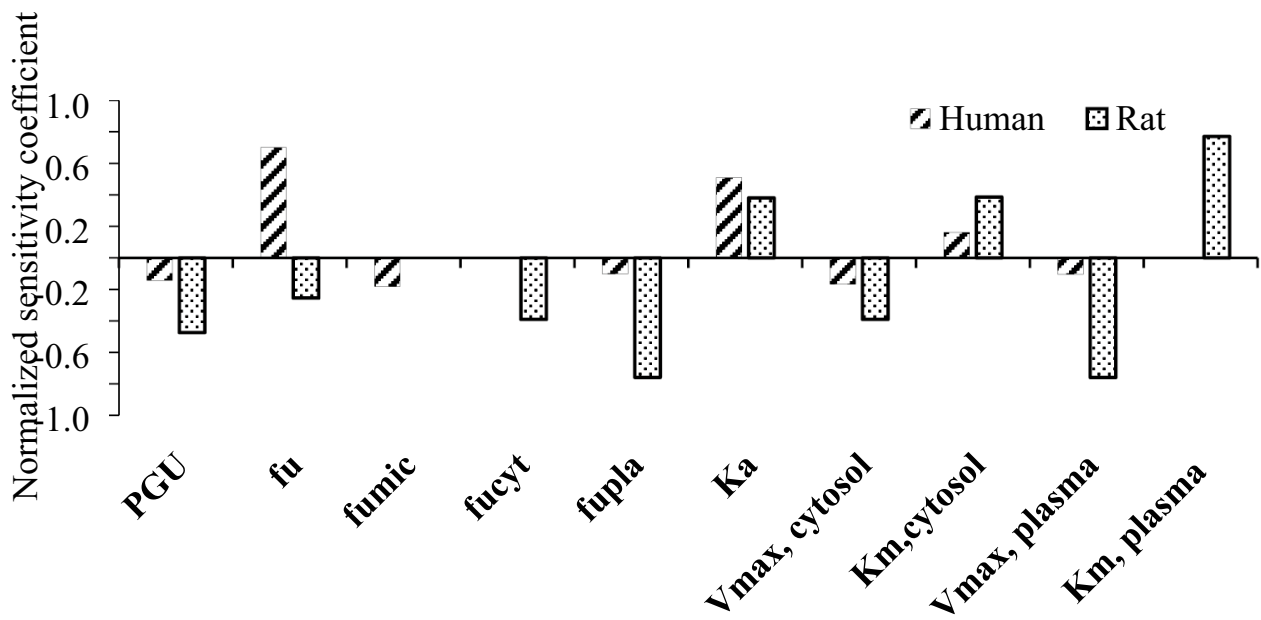

Fig. 5 Sensitivity analysis representing the influence of model parameters on the predicted blood $C_{\max }$ of profenofos in humans and rats at predicted $\mathrm{BMDL}_{10}$ values of 0.01 and $0.45 \mathrm{mg} / \mathrm{kg}$ bw, respectively. $P G U$ gut:plasma partition coefficient, $f u$ fraction unbound in plasma, fumic fraction unbound in microsomal incubation, fucyt fraction unbound in cytosolic incubation, fupla fraction unbound in incuba-

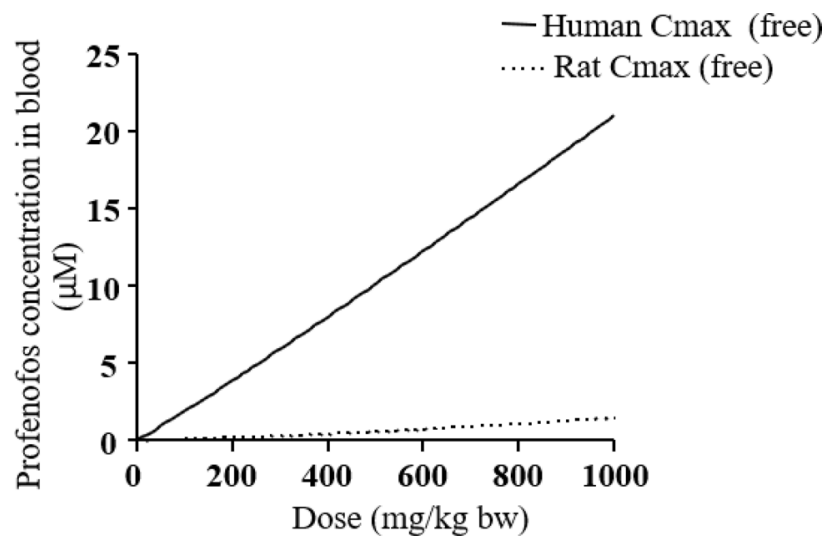

Fig. 6 PBK model-predicted dose-dependent $C_{\max }$ (free) in blood upon oral profenofos exposure in rats (dotted line) and humans (continuous line)

\section{Prediction of in vivo dose-dependent $\mathrm{AChE}$ inhibition by PBK modeling-facilitated reverse dosimetry of in vitro data and comparison with reported in vivo effect data}

Concentration-dependent inhibition of rat $\mathrm{RBC} \mathrm{AChE}$ (obtained from rat blood) and human (recombinant) $\mathrm{AChE}$ by profenofos were obtained in vitro (Fig. 7). Profenofos inhibited human AChE with an IC50 value of $302 \mathrm{nM}$, which is very close to the IC50 value of $350 \mathrm{nM}$ reported by Das and Jamil (2006) using freshly obtained human erythrocytes. Profenofos inhibited rat AChE with an IC50 of $312 \mathrm{nM}$, suggesting no species difference in profenofos-induced $\mathrm{AChE}$ tion with plasma, $k a$ absorption constant, $V_{\max }$, cytosol=maximum rate of conversion of profenofos to BCP by liver cytosol, $\mathrm{Km}$ cytosol Michaelis-Menten constant for conversion of profenofos to BCP by liver cytosol, $V_{\max }$ plasma = maximum rate of conversion of profenofos to BCP by plasma, $K m$ plasma= Michaelis-Menten constant for conversion of profenofos to BCP by plasma

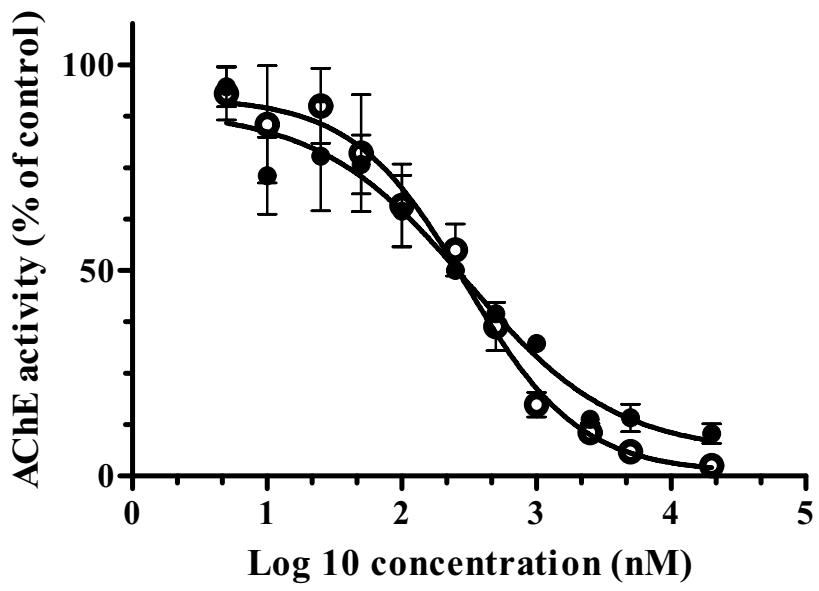

Fig. 7 Acetylcholinesterase (AChE) activity in RBCs of SpragueDawley rats (closed symbols) and of human recombinant AChE (open symbols) with increasing concentrations of profenofos. AChE activity in the solvent control is set at $100 \%$. Results represent data from 3 independent experiments and are presented as mean \pm SEM

inhibition, based on effect concentrations related to $50 \%$ AChE inhibition.

The concentration-dependent AChE inhibition curves obtained in this study were converted into in vivo dosedependent AChE inhibition curves in rats and humans (Fig. 8) using the PBK modeling-facilitated reverse dosimetry approach as described in the materials and methods section. Figure 8 also presents in vivo data for male and female rat profenofos-induced AChE inhibition in RBCs and in the brain available from literature (JMPR 2007). The 
results reveal that predicted in vivo dose-response curves for rat $\mathrm{AChE}$ inhibition are close to the reported in vivo data for male and female rat AChE inhibition in RBCs and in the brain (JMPR 2007), indicating that with this combined in vitro-in silico approach, good predictions for rats were obtained. Predictions for humans indicate that humans are expected to be more sensitive than rats regarding profenofosinduced AChE inhibition (Fig. 8), which is mainly due to the slower metabolic clearance of profenofos, resulting in higher $C_{\max }$ values at the same dose levels (Fig. 6). Toxicity data for humans for further evaluation of the predicted dose-response curves are not available.

BMD analysis was performed on predicted dose-response data for rats and human. Results of these analyses are presented in Supplementary Tables 2 and 3 and Supplementary Figs. 2 and 3. BMDL 10 values obtained for rats and humans upon model averaging were 0.45 and $0.01 \mathrm{mg} / \mathrm{kg}$ bw, respectively, predicting humans to be more sensitive than rats. In theory, BMD values obtained from predicted dose-response data may serve as PODs for setting safe exposure levels, i.e., an ARfD, and these BMD values were, therefore, compared with PODs that have been used by the US EPA, JMPR and EFSA in their assessments.

The US EPA considered erythrocyte (RBC) AChE inhibition to be more suitable to derive a POD for human safety assessments, since it is more sensitive than brain AChE inhibition in case of profenofos exposure (EPA 1999, 2016). A $10 \%$ RBC inhibition of AChE has been adopted by the US EPA to obtain a POD to define the ARfD for profenofos

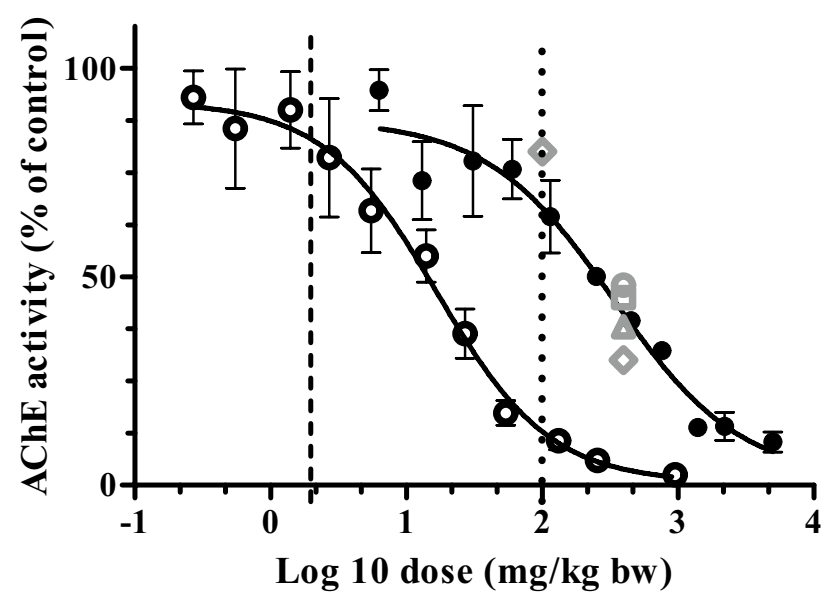

Fig. 8 Predicted dose-response curves for profenofos-induced decrease of AChE activity in rats (closed black circles) and humans (open black circles). In vivo data on profenofos-induced AChE inhibition in rats as reported in JMPR (2007) are included: open grey diamond-male rat RBC inhibition, open grey triangle-male rat brain inhibition, open grey square-female rat brain inhibition, open grey circle-female rat RBC inhibition. EPA BMDL 10 and JMPR NOAEL are indicated by the vertical lines (EPA 2016: dashed line; JMPR 2007: dotted line)
(EPA 2016). The US EPA reported a $\mathrm{BMDL}_{10}$ of $1.99 \mathrm{mg} /$ $\mathrm{kg}$ bw for profenofos-induced RBC AChE inhibition in adult rats upon a single exposure to profenofos, being 4 times higher than the $\mathrm{BMDL}_{10}$ obtained from the predicted dose-response data for rats in our study $(0.45 \mathrm{mg} / \mathrm{kg} \mathrm{bw})$.

JMPR used a NOAEL value of $100 \mathrm{mg} / \mathrm{kg}$ bw to derive an ARfD, based on a dataset on profenofos-induced inhibition of brain $\mathrm{AChE}$ in rats (upon a single exposure to profenofos). This NOAEL value is 50 -fold higher than the $\mathrm{BMDL}_{10}$ value reported by the US EPA and 200-fold higher than the $\mathrm{BMDL}_{10}$ value obtained from our predicted dose-response data in rats $(0.45 \mathrm{mg} / \mathrm{kg} \mathrm{bw})$, suggesting the NOAEL to result in a POD that may be too high.

In a recent report from EFSA (EFSA 2019), an ARfD for profenofos of $0.005 \mathrm{mg} / \mathrm{kg}$ bw was reported, based on a German evaluation in 2001 that defined an ARfD based on a NOAEL in a dog study using inhibition of brain cholinesterase activity as critical effect. Unfortunately, no further information (e.g., NOAEL) on that particular dog study is publicly available. If a safety factor of 100 was used to derive this ARfD, the NOAEL derived from the dog study would be close to the $\mathrm{BMDL}_{10}$ value obtained from our predicted dose-response data in rats $(0.45 \mathrm{mg} / \mathrm{kg}$ bw ( 100-fold higher than the ARfD)).

\section{Discussion}

The aim of the current study was to develop a physiologically based kinetic (PBK) model in rats and humans solely based on in vitro and in silico input parameters to be applied for the prediction of in vivo AChE inhibition by the OP pesticide profenofos by reverse dosimetry of in vitro AChE inhibition data. To this end, profenofos PBK models were developed for rats and humans and used to predict dosedependent internal profenofos concentrations, which were applied for reverse dosimetry to translate in vitro concentration-dependent data on profenofos-induced inhibition of AChE to in vivo dose-response curves for profenofosinduced inhibition of AChE in blood. The results indicate that profenofos-induced $\mathrm{AChE}$ inhibition in rats was closely predicted and that humans are predicted to be more sensitive to profenofos-induced $\mathrm{AChE}$ inhibition than rats upon acute exposure.

This study has shown marked interspecies differences in toxicokinetics of profenofos with predicted blood $C_{\max }$ values for profenofos in humans being around 20-fold higher than in rats at equal levels of exposure (Fig. 6). This is mainly due to higher catalytic efficiencies for microsomal, cytosolic and plasma biotransformation of profenofos to BCP in rats as compared to humans as observed in this study (Table 1). Profenofos, being an oxon, is mainly detoxified through hydrolysis by hepatic and plasma PON1 and 
CYP450-mediated detoxification, resulting in the production of BCP (JMPR 2007; EPA 2016; Dadson et al. 2013). Interspecies differences in activities between human and rat PON1 have been reported, with human PON1 having lower catalytic activity as compared to rat PON1 (KalisteKorhonen et al. 1996). Another possible reason for the differences in profenofos detoxification may be related to quantitative differences in B-esterases in rats and humans, which may affect the metabolism and disposition of ester compounds including OPs (Ecobichon and Comeau 1973; Maxwell et al. 1987). B-esterases, such as carboxylesterase $(\mathrm{CaE})$, butyrylcholinesterase $(\mathrm{BuChE})$ and acetylcholinesterase (AChE), detoxify oxons, but these enzymes are inhibited by the oxons as a consequence (Chanda et al. 1997). It has been reported that rat plasma contains almost all types of esterases including $\mathrm{CaE}, \mathrm{PON} 1, \mathrm{BChE}$, and $\mathrm{AChE}$ (Bahar et al. 2012; Satoh and Hosokawa 2006), whereas humans are deficient of plasma CaE (Williams et al. 2011; Berry et al. 2009; Li et al. 2005) and plasma AChE (Ecobichon and Comeau 1973). The relevance of $\mathrm{CaE}$ in OP detoxification is indicated by a study that demonstrated that in vivo inhibition of CaEs by cresylbenzodioxaphosphorin oxide (CBDP) caused an increase in the toxicity of many OPs in rats, mice, rabbits and guinea pigs (Ecobichon and Comeau 1973; Maxwell et al. 1987). In the same study, the interspecies differences observed in the toxicity of OPs were lost in the group treated with the in vivo $\mathrm{CaE}$ inhibitor (CBDP) (Maxwell et al. 1987). The absence of $\mathrm{CaE}$ in human plasma in addition to low PON1 activity may, therefore, be responsible for lower profenofos clearance resulting in higher sensitivity of humans to profenofos as compared to rodents (KalisteKorhonen et al. 1996). In this way, B-esterases may play a protective role in rats but not humans.

Data on RBC AChE inhibition can be considered as an appropriate surrogate measure of potential organophosphate effects on the peripheral and central nervous systems in absence of brain AChE inhibition data (Chen et al. 1999; Das and Jamil 2006). To predict profenofos dose levels that result in AChE inhibition in rats and humans, profenofos in vitro concentration-response curves for RBC AChE inhibition (Fig. 7) were translated to in vivo dose-response curves for RBC AChE inhibition for both rats and humans (Fig. 8). The predicted data thus obtained for the rat matched with the available in vivo rat data as reported by JMPR (2007) (Fig. 8), and the $\mathrm{BMDL}_{10}$ obtained from the predicted dose-response data differed only fourfold from the $\mathrm{BMDL}_{10}$ obtained from data from an in vivo rat study on profenofos-induced blood $\mathrm{AChE}$ inhibition used by the US EPA for determination of an ARfD for profenofos. This indicates that in vivo dose-dependent AChE inhibition was closely predicted based on our approach, giving confidence in the human predictions, which could not be further evaluated because of the lack of human in vivo data on this endpoint. Our predictions suggest that humans are more sensitive than rats towards profenofos-induced $\mathrm{AChE}$ inhibition. The interspecies differences (45-fold difference in $\mathrm{BMDL}_{10}$ ) were predicted to be mainly due to interspecies differences in toxicokinetics, as discussed above, and these would not be covered by the standard uncertainty factor of 10 to account for interspecies differences. Currently, we work on the assessment of human interindividual differences in profenofos metabolic clearance (using plasma and liver fractions of different donors) to be incorporated in PBK models, providing insight into whether a combined uncertainty factor of 100 (10 for interspecies differences and 10 for intraspecies differences) applied to the rat $\mathrm{AChE}$ inhibition data is expected to be sufficiently protective for sensitive human individuals.

In the present study, the $\mathrm{BMDL}_{10}$ calculated based on predicted rat data was 4 times lower than the $\mathrm{BMDL}_{10}$ value used by EPA and 200-fold lower than the NOAEL used by JMPR to obtain an ARfD, implying that our prediction is more conservative as compared to the rat data used by the two agencies. In a possible future risk assessment paradigm that would be independent of animal data, $\mathrm{BMDL}_{10}$ values obtained from the predicted human dose-response data may be applied. In the present study, we determined a $\mathrm{BMDL}_{10}$ value for 'an average human' $(0.01 \mathrm{mg} / \mathrm{kg} \mathrm{bw})$, not quantifying possible interindividual differences. To take interindividual differences into account, an uncertainty factor may be applied, but it would be scientifically more sound to quantify interindividual differences in the human population in profenofos detoxification. This is especially of interest given the reported genetic polymorphisms of human PON1 (and BuChE) with related phenotypes of low and high activities (Schwarz et al. 1995; Geldmacher et al. 1989), which may play a role in the interindividual differences in PON1 activity (Darney et al. 2020), suggesting possible large human interindividual differences in profenofos detoxification. As indicated above, we currently work on the determination of such interindividual differences in profenofos detoxification in humans and integrate these data in the human PBK modeling-based predictions of profenofos toxicity.

Although the current models quantitatively predicted profenofos tissue dosimetry and the resulting $\mathrm{AChE}$ inhibition, possible limitations of the approach should be considered. In the current PBK model, gut absorption of profenofos was estimated to be $100 \%$ using a first-order process with an absorption rate constant of $1 \mathrm{~h}^{-1}$. Although this resulted in the adequate prediction of time-dependent $\mathrm{BCP}$-glucuronide in rat urine, it must be noted that that specific model output is not so sensitive to the ka (data not shown), whereas the (unbound) $C_{\max }$ is (Fig. 5). It is noteworthy that the extent of absorption varies depending on the dosing method, dose formulation (solution) and variations in species, strain and 
gender (Kararli 1995). Furthermore, profenofos absorption as present in the food matrix may be different.

We used a static approach to determine the AChE inhibitory effect concentrations, using a single pre-incubation time point $(15 \mathrm{~min})$. When including more time points, information on the time-dependent inhibition kinetics can be obtained. However, a previous study with chlorpyrifos oxon showed that human recombinant AChE active sites were $100 \%$ inhibited after a pre-incubation period of $11 \mathrm{~min}$ (Kaushik et al. 2007). One would expect that effect concentrations would decrease with longer incubation times until $100 \%$ of the binding sites is inhibited, as shown before by Aurbek et al. (2006) and Krstić et al. (2008). With a relatively long pre-incubation time of $15 \mathrm{~min}$ as we have used in the present study, similar to incubation times previously applied by other research groups (e.g., Aurbek et al. 2006; Kasteel et al. 2020), we aimed to obtain a relevant and conservative estimation of the effect concentrations, as also indicated by the small difference between the $\mathrm{BMDL}_{10}$ value obtained with our predicted rat dose-response data and the $\mathrm{BMDL}_{10}$ value from a reported in vivo study in rats.

It must also be noted that the approach used in this study may be suitable to estimate AChE inhibition upon a single exposure, while not predicting effects upon repeated exposure, since upon longer exposures, one must take the time-dependent de novo $\mathrm{AChE}$ production into account to adequately estimate the $\mathrm{AChE}$ activity upon a second and further exposure. In that regard, a phenomenon referred to as steady state $\mathrm{AChE}$ inhibition is of interest, which is the situation when the degree of AChE inhibition reaches equilibrium with the production of new enzyme at which AChE inhibition remains constant at a specified dose over the exposure period (EPA 2016). Adequate prediction of these processes based on only in vitro data, as applied in the present study, seems at this stage not possible. Furthermore, one must consider that OPs inhibit detoxifying enzymes, such as $\mathrm{CaE}$ and $\mathrm{BuChe}$, which may result in a decrease in metabolic clearance, and related higher internal concentrations and increased sensitivity upon repeated dosing.

Another limitation of the approach is that the prediction of OP toxicity was only based on AChE inhibition as the endpoint. It should, however, be noted that OPs may affect a number of additional targets (such as Neuropathy target esterase (NTE) (Costa 2018), muscarinic M2 receptors (Costa 2006), acylpeptide hydrolase (APH) (Richards et al. 2000), fatty acid amide hydrolase (FAAH) (Quistad et al. 2001; Buntyn et al. 2017) and a variety of lipases (Quistad et al. 2006)) that lead to OP toxicity, including neuroinflammation, autoimmunity and axonal transport deficits (Naughton and Terry 2018). On the other hand, AChE inhibition has been used as an endpoint to set a POD for the risk assessment (EPA 2016; EFSA 2019), indicating its relevance. The QIVIVE approach could be extended in the future to also predict dose-dependent effects related to other OP targets, allowing a more extensive hazard assessment based on NAMs.

In conclusion, the predicted dose-dependent profenofosinduced $\mathrm{AChE}$ inhibition in rats are close to reported data on dose-dependent in vivo $\mathrm{AChE}$ inhibition in rats upon single dosing, providing also confidence in the predictions obtained for humans. Results from this study suggest that humans may be more sensitive to AChE inhibition upon profenofos exposure than rats, which is mainly due to differences in profenofos detoxification. Altogether, the results demonstrate the ability to predict in vivo AChE inhibition by profenofos, providing another proof-of-principle that with NAMs in vivo effects of chemicals can be predicted without the use of in vivo studies.

Supplementary Information The online version contains supplementary material available at https://doi.org/10.1007/s00204-021-03004-4.

Acknowledgements This work is supported by the Netherlands Universities Foundation for International Cooperation (Nuffic) [scholarship granted to Isaac Omwenga (PhD.17/0019)], the China Scholarship Council [scholarship (No. 201707720063) Granted to Shensheng Zhao], and the Dutch Ministry of Agriculture, Nature and Food Quality (project KB-23-002-021).

\section{Compliance with ethical standards}

Conflict of interest The authors declare that they have no conflicts of interest.

Open Access This article is licensed under a Creative Commons Attribution 4.0 International License, which permits use, sharing, adaptation, distribution and reproduction in any medium or format, as long as you give appropriate credit to the original author(s) and the source, provide a link to the Creative Commons licence, and indicate if changes were made. The images or other third party material in this article are included in the article's Creative Commons licence, unless indicated otherwise in a credit line to the material. If material is not included in the article's Creative Commons licence and your intended use is not permitted by statutory regulation or exceeds the permitted use, you will need to obtain permission directly from the copyright holder. To view a copy of this licence, visit http://creativecommons.org/licenses/by/4.0/.

\section{References}

Aly OA, Badawy MI (1982) Hydrolysis of organophosphate insecticides in aqueous media. Environ Int 7:373-377

Aurbek N, Thiermann H, Szinicz L, Eyer P, Worek F (2006) Analysis of inhibition, reactivation and aging kinetics of highly toxic organophosphorus compounds with human and pig acetylcholinesterase. Toxicology 224:91-99

Bahar FG, Ohura K, Ogihara T, Imai T (2012) Species difference of esterase expression and hydrolase activity in plasma. J Pharm Sci 101:3979-3988 
Berezhkovskiy LM (2004) Volume of distribution at steady state for a linear pharmacokinetic system with peripheral elimination. J Pharm Sci 93:1628-1640

Berry LM, Wollenberg L, Zhao Z (2009) Esterase activities in the blood, liver and intestine of several preclinical species and humans. Drug Metab Lett 3:70-77

Bogen KT, Singhal A (2017) Malathion dermal permeability in relation to dermal load: Assessment by physiologically based pharmacokinetic modeling of in vivo human data. J Environ Sci Health B 52:138-146

Bouchard M, Gosselin NH, Brunet RC, Samuel O, Dumoulin MJ, Carrier G (2003) A toxicokinetic model of malathion and its metabolites as a tool to assess human exposure and risk through measurements of urinary biomarkers. Toxicol Sci 73:182-194

Bouchard M, Carrier G, Brunet RC, Bonvalot Y, Gosselin NH (2005) Determination of biological reference values for chlorpyrifos metabolites in human urine using a toxicokinetic approach. J Occup Environ Hyg 2:155-168

Bouchard M, Gosselin NH, Brunet RC, Samuel O, Dumoulin M, Carrier G (2017) A toxicokinetic model of malathion and its metabolites as a tool to assess human exposure and risk through measurements of urinary biomarkers. Toxicol Sci 73:182-194

Bradman A, Barr DB, Claus-Henn BG, Drumheller T, Curry C, Eskenazi B (2003) Measurement of pesticides and other toxicants in amniotic fluid as a potential biomarker of prenatal exposure: a validation study. Environ Health Perspect 111:1779-1182

Buntyn RW, Alugubelly N, Hybart RL, Mohammed AN, Nail CA, Parker GC, Ross MK, Carr RL (2017) Inhibition of endocannabinoid-metabolizing enzymes in peripheral tissues following developmental chlorpyrifos exposure in rats. Int J Toxicol 36:395-402

Carr RL, Dail MB, Chambers HW, Chambers JE (2015) Species differences in paraoxonase mediated hydrolysis of several organophosphorus insecticide metabolites. J Toxicol 470189

Chambers JE, Chambers JW (1989) Oxidative desulfuration of chlorpyrifos, chlorpyrifos-methyl and leptophos by rat brain and liver. J Biochem Toxicol 4:201-203

Chanda SM, Mortensen SR, Moser VC, Padilla S (1997) Tissue-specific effects of chlorpyrifos on carboxylesterase and cholinesterase activity in adult rats: an in vitro and in vivo comparison. Fundam Appl Toxicol 38:148-157

Chen WL, Sheets JJ, Nolan RJ, Mattsson JL (1999) Human red blood cell acetylcholinesterase inhibition as the appropriate and conservative surrogate endpoint for establishing chlorpyrifos reference dose. Regul Toxicol Pharmacol 29:15-22

Cho Y, Min K, Lee I, Cha C (2002) Determination of urinary metabolite of profenofos after oral administration and dermal application to rats. J Food Hyg Saf 17:20-25

Costa LG (2006) Current issues in organophosphate toxicology. Clin Chim Acta 366:1-13

Costa LG (2018) Organophosphorus compounds at 80: some old and new issues. Toxicol Sci 162:24-35

Cubitt HE, Houston JB, Galetin A (2011) Prediction of human drug clearance by multiple metabolic pathways: integration of hepatic and intestinal microsomal and cytosolic data. Drug Metab Dispos 39:864-873

Dadson OA, Ellison CA, Singleton ST, Chi L, McGarrigle BP, Pamela J, Lein JP, Farahat FM, Farahat T, Olson JR (2013) Metabolism of profenofos to 4-bromo-2-chlorophenol, a specific and sensitive exposure biomarker. Toxicology 306:35-39

Darney K, Kasteel EEJ, Buratti FM, Turco L, Vichi S, Béchaux C, Roudot AC, Kramer NI, Testai E, Dorne JLCM, Di Consiglio E, Lautz LS (2020) Bayesian meta-analysis of inter-phenotypic differences in human serum paraoxonase-1 activity for chemical risk assessment. Environ Int 138:105609
Das GP, Jamil K (2006) Effect of four organophosphorus compounds on human blood acetylcholinesterase. Vitro Stud Toxicol Mech Methods 16:455-459

Ecobichon DJ, Comeau AM (1973) Pseudocholinesterases of mammalian plasma: Physicochemical properties and organophosphate inhibition in eleven species. Toxicol Appl Pharmacol 24:92-100

Eddleston M, Worek F, Eyer P, Thiermann H, Von Meyer L, Jeganathan K, Sheriff MHR, Dawson AH, Buckley NA (2009) Poisoning with the S-Alkyl organophosphorus insecticides profenofos and prothiofos. QJM Int J Med 102:785-792

Ellman GL, Courtney KD, Andres V, Featherstone RM (1961) A new rapid colorimetric determination of acetylcholinesterase activity. Biochem Pharmacol 7:88-95

EFSA (European Food Safety Authority) (2019) Scientific report on scientific support for preparing an EU position in the 51st Session of the Codex Committee on Pesticide Residues (CCPR). EFSA J 17:5797

EPA (1999) Data evaluation record study type: special non-guideline assessment for RBC Cholinesterase in humans. https://archi ve.epa.gov/scipoly/sap/meetings/web/pdf/kisickider.pdf Accessed Jan 2019

EPA (2006) Reregistration eligibility decision for dimethoate. https:// archive.epa.gov/pesticides/reregistration/web/pdf/dimethoate_red. pdf. Accessed Dec 2020

EPA (2011) Chlorpyrifos preliminary human health risk assessment DP No. D388070. https://www.regulations.gov/contentStreamer ?documentId=EPA-HQ-OPP-2008-0850-0025\&contentTyp $\mathrm{e}=$ pdf. Accessed Dec 2020

EPA (2016) Profenofos: Human health draft risk assessment (DRA) for registration review.https://www3.epa.gov/pesticides/chem_searc h/cleared_reviews/csr_PC-111401_19-Oct-16.pdf. Accessed Mar 2020

Evans MV, Andersen ME (2000) Sensitivity analysis of a physiological model for 2, 3, 7, 8-tetrachlorodibenzo- $p$-dioxin (TCDD): assessing the impact of specific model parameters on sequestration in liver and fat in the rat. Toxicol Sci 54:71-80

Farahat TM, Abdelrasoul GM, Amr MM, Shebl MM, Farahat FM, Anger WK (2003) Neurobehavioral effects among workers occupationally exposed to organophosphorous pesticides. Occup Environ Med 60:279-286

Geldmacher-v M, Diepgen TL (1989) Human serum paraoxonase polymorphism, specificity, classification. In: Reiner E, Aldridge WN, Hoskin FCG (eds) Enzymes hydrolyzing organophosphorus compounds Ellis Horwood Limited. West Sussex, England, pp 15-29

Gotoh M, Sakata M, Endo T, Hayashi H, Seno H, Suzuki O (2001) Profenofos metabolites in human poisoning. Forensic Sci Int 116:221-226

Greish S, Ismail SM, Mosleh Y, Loutfy N, Dessouki AA, Ahmed MT (2011) Human risk assessment of profenofos: a case study in Ismailia. Egypt Polycycl Aromat Compd 31:28-47

Hardt J, Angerer J (2000) Determination of dialkyl phosphates in human urine using gas chromatography-mass spectrometry. J Anal Toxicol 24:678-684

Heilmair R, Eyer F, Eyer P (2008) Enzyme-based assay for quantification of chlorpyrifos oxon in human plasma. Toxicol Lett 181:19-24

Hertz-Picciotto I, Sass JB, Engel S, Bennett DH, Bradman A, Eskenazi B, Lanphear B, Whyatt B (2018) Organophosphate exposures during pregnancy and child neurodevelopment: recommendations for essential policy reforms. PLoS Med 15(10):e1002671

Jamal GA, Hansen S, Julu POO (2002) Low level exposures to organophosphorus esters may cause neurotoxicity. Toxicology 181-182:23-33

Johnson MK, Glynn P (1995) Neuropathy target esterase (NTE) and 675 organophosphorus induced delayed polyneuropathy (OPIDP): recent 676 advances. Toxicol Lett 82-83:459-463 
Johnson MK, Glynn P (2001) Neuropathy target esterase. In: Krieger R (ed) Handbook of pesticide toxicology. Academic Press, San Diego, pp 953-965

Joint FAO/WHO Meeting on Pesticide Residues (JMPR) (2007) Pesticide residues in food 2007. http://www.inchem.org/documents/ jmpr/jmpmono/v2007pr01.pdf. Accessed Aug 2020

Jones HM, Rowland-Yeo K (2013) Basic concepts in physiologically based pharmacokinetic modeling in drug discovery and development. CPT Pharmacometr Syst Pharmacol 2:e63

Kaliste-Korhonen E, Tuovinen K, Hänninen O (1996) In vitro interspecies differences in enzymes reacting with organophosphates and their inhibition by paraoxon. Hum Exp Toxicol 15:972

Karanja NK, Njenga M, Mutua GK, Lagerkvist CJ, Kutto E, Okello JJ (2012) Concentrations of heavy metals and pesticide residues in leafy vegetables and implications for peri-urban farming in Nairobi, Kenya. J Agric Food Syst Commun Dev 3:255-267

Kararli TT (1995) Comparison of the gastrointestinal anatomy, physiology and biochemistry of humans and commonly used laboratory animals. Biopharm Drug Dispos 16:351-380

Kasteel EEJ, Nijmeijer SM, Darney K, Lautz LS, Dorne JLCM, Kramer NI, Westerink RHS (2020) Acetylcholinesterase inhibition in electric eel and human donor blood: an in vitro approach to investigate interspecies differences and human variability in toxicodynamics. Arch Toxicol 94:4055-4065

Kaushik R, Rosenfeld CA, Sultatos LG (2007) Concentration-dependent interactions of the organophosphates chlorpyrifos oxon and methyl paraoxon with human recombinant acetylcholinesterase. Toxicol Appl Pharmacol 221:243-250

Krstić DZ, Colović M, Kralj MB, Franko M, Krinulović K, Trebse P, Vasić V (2008) Inhibition of AChE by malathion and some structurally similar compounds. J Enzyme Inhib Med Chem 23:562-573

Kumari D, John S (2019) Health risk assessment of pesticide residues in fruits and vegetables from farms and markets of Western Indian Himalayan Region. Chemosphere 224:162-167

Larsen KE, Lifschitz AL, Lanusse CE, Virkel GL (2019) In vitro and in vivo effects of chlorpyrifos and cypermethrin on blood cholinesterases in sheep. J Vet Pharmacol Therap 42:548-555

Li B, Sedlacek M, Manoharan I, Boopathy R, Duysen EG, Masson P, Lockridge O (2005) Butyrylcholinesterase, paraoxonase, and albumin esterase, but not carboxylesterase, are present in human plasma. Biochem Pharmacol 70:1673-1684

Liu P, Wu CH, Chang XL, Qi XJ, Zheng ML, Zhou ZJ (2014) Assessment of chlorpyrifos exposure and absorbed daily doses among infants living in an agricultural area of the Province of Jiangsu, China. Int Arch Occup Environ Health 87:753-762

Louisse J, Beekmann K, Rietjens IMCM (2017) Use of physiologically based kinetic modeling-based reverse dosimetry to predict in vivo toxicity from in vitro data. Chem Res Toxicol 30:114-125

Lowry O, Rosebrough N, Farr A, Randall R (1951) Protein measurement with the folin phenol reagent. J Biol Chem 193:265-275

Lu C, Fenske RA, Simcox NJ, Kalman D (2000) Pesticide exposure of children in agricultural community: evidence of household proximity to farmland and take home exposure pathways. Environ Res Sect A 84:290-302

Lu C, Holbrook CM, Andres LM (2010) The implications of using a physiologically based pharmacokinetic (PBPK) model for pesticide risk assessment. Environ Health Perspect 118:125-130

Maxwell DM, Lenz DE, Groff WA, Kaminskis A, Froehlich HL (1987) The effects of blood flow and detoxification on in vivo cholinesterase inhibition by soman in rats. Toxicol Appl Pharmacol 88:66-76

Medinsky MA, Leavens TL, Csanady GA, Gargas ML, Bond JA (1994) In vivo metabolism of butadiene by mice and rats: a comparison of physiological model predictions and experimental data. Carcinogenesis 15:1329-1340
Mosquin PL, Licata AC, Liu B, Sumner SC, Okino MS (2009) Reconstructing exposures from small samples using physiologically based pharmacokinetic models and multiple biomarkers. J Expo Sci Environ Epidemiol 19:284-297

Mostafalou S, Abdollahi M (2013) Pesticides and human chronic diseases: evidences, mechanisms, and perspectives. Toxicol Appl Pharmacol 268:157-177

Naksen W, Prapamontol T, Mangklabruks A, Chantara S, Thavornyutikarn P, Robson MG, Panuwet P (2016) A single method for detecting 11 organophosphate pesticides in human plasma and breastmilk using GC-FPD. J Chromatogr B Analyt Technol Biomed Life Sci 1025:92-104

Naughton SX, Terry AV Jr (2018) Neurotoxicity in acute and repeated organophosphate exposure. Toxicology 408:101-112

Omwenga I, Kanja L, Zomer P, Louisse J, Rietjens IMCM, Mol H (2021) Organophosphate and carbamate pesticide residues and accompanying risks in commonly consumed vegetables in Kenya. Food Addit Contam Part B 14:48-58

Poet TS, Kousba AA, Dennison SL, Timchalk C (2004) Physiologically based pharmacokinetic/pharmacodynamic model for the organophosphorus pesticide diazinon. Neurotoxicology 25:1013-1030

Punt A, Pinckaers N, Peijnenburg A, Louisse J (2021) Development of a web-based toolbox to support quantitative in vitro-to-in vivo extrapolations (QIVIVE) within non-animal testing strategies. Chem Res Toxicol 34:460-472

Quandt SA, Arcury TA, Rao P, Snively BM, Camann DE, Doran AM, Yau AY, Hoppin JA, Jackson DS (2004) Agricultural and residential pesticides in wipe samples from farmworker family residences in North Carolina and Virginia. Environ Health Perspect 112:382-387

Quistad GB, Sparks SE, Casida JE (2001) Fatty acid amide hydrolase inhibition by neurotoxic organophosphorus pesticides. Toxicol Appl Pharmacol 173:48-55

Quistad GB, Liang SN, Fisher KJ, Nomura DK, Casida JE (2006) Each lipase has a unique sensitivity profile for organophosphorus inhibitors. Toxicol Sci 91:166-172

Richards PG, Johnson MK, Ray DE (2000) Identification of acylpeptide hydrolase as a sensitive site for reaction with organophosphorus compounds and a potential target for cognitive enhancing drugs. Mol Pharmacol 58:577-583

Rigas ML, Okino MS, Quackenboss JJ (2001) Use of a pharmacokinetic model to assess chlorpyrifos exposure and dose in children, based on urinary biomarker measurements. Toxicol Sci 61:374-381

Rosenfeld CA, Sultatos LG (2006) Concentration-dependent kinetics of acetylcholinesterase inhibition by the organophosphate paraoxon. Toxicol Sci 90:460-469

Satoh T, Hosokawa M (2006) Structure, function and regulation of carboxylesterases. Chem Biol Interact 162:195-211

Schwarz M, Loewenstein-Lichtenstein Y, Glick D, Liao J, NorgaardPedersen B, Soreq H (1995) Successive organophosphate inhibition and oxime reactivation reveals distinct responses of recombinant human cholinesterase variants. Brain Res Mol Brain Res $31: 101-110$

Strikwold M, Spenkelink B, Woutersen R, Rietjens IMCM, Punt A (2013) Combining in vitro embryotoxicity data with physiologically based kinetic (PBK) modelling to define in vivo dose response curves for developmental toxicity of phenol in rat and human. Arch Toxicol 87:1709-1723

Sultatos LG (1990) A physiologically based pharmacokinetic model of parathion based on chemical-specific parameters determined in vitro. J Am Coll Toxicol 9:611-619

Timchalk C, Nolan RJ, Mendrala AL, Dittenber DA, Brzak KA, Mattsson JL (2002) A physiologically based pharmacokinetic and pharmacodynamic (PBPK/PD) model for the organophosphate insecticide chlorpyrifos in rats and humans. Toxicol Sci 66:34-53 
Williams ET, Bacon JA, Bender DM, Lowinger JJ, Guo WK, Ehsani ME, Wang X, Wang H, Qian YW, Ruterbories KJ, Wrighton SA, Perkins EJ (2011) Characterization of the expression and activity of carboxylesterases 1 and 2 from the beagle dog, cynomolgus monkey, and human. Drug Metab Dispos 39:2305-2313

Zhang Y, Han S, Liang D, Shi X, Wang F, Liu W, Zhang L, Chen L, Gu Y, Tian Y (2014) Prenatal exposure to organophosphate pesticides and neurobehavioral development of neonates: a birth cohort study in Shenyang. China PloS one 9:88491

Zhao S, Kamelia L, Boonpawa R, Wesseling S, Spenkelink B, Rietjens IMCM (2019) Physiologically based kinetic modelling-facilitated reverse dosimetry to predict in vivo red blood cell acetylcholinesterase inhibition following exposure to chlorpyrifos in the Caucasian and Chinese population. Toxicol Sci 171:69-83

Publisher's Note Springer Nature remains neutral with regard to jurisdictional claims in published maps and institutional affiliations. 